\title{
New Insight into Acute Myeloid Leukemia Classification and Risk Stratification
}

\author{
Nahla AM Hamed* \\ Faculty of Medicine, Alexandria University, Egypt
}

Submission: June 12, 2018; Published: July 03, 2018

"Correspondence Address: Nahla AM Hamed, Professor of Hematology, Faculty of Medicine, Alexandria University, Egypt,

Email: drhamedn@hotmail.com

Abstract

AML is the most commonly occurring acute hematological malignancy in adults. It represents more than $80 \%$ of all leukemias in patients over 60 years of age. The probability of acquiring a mutation in a gene that will confer selective advantage and clonal selection in hematopoietic stem and progenitor cells increases with age. The focus of this review is to highlight on what is new in the 2016 revision to the World Health Organization classification of AML and related neoplasms. Attention to AML risk status by cytogenetics and molecular abnormalities will also be summarized.

Abbrevations: AML: Acute Myeloid Leukemia; FLT3: Fms-related Tyrosine Kinase 3; ITD: Internal Tandem Duplication; APL: Acute Promyelocytic Leukemia; BM: Bone Marrow; TAM: Transient Abnormal Myelopoiesis; MPAL: Acute Leukemias of Ambiguous Lineage; BPDCN: Blastic Plasmacytoid Dendritic Cell Neoplasm; NPM1: Nucleophosmin; DNMT3A: DNA Methyltransferase 3A; NK: Normal Karyotyping

\section{Introduction}

At the time of AML diagnosis, prognostically important and distinct chromosomal translocations and large chromosome gains or losses can be observed on standard karyotype analysis in approximately half of AML patients [1]. NK-AML accounts for $50 \%$ of AML. This category segregates in distinct and nonoverlapping mutational groups: NPM1 mutated, bi-allelic CEBPA, and the chromatin-spliceosome group [2]. The identification that CEBPA, NPM1, and FLT3- ITD mutations predict response to induction and consolidation chemotherapy for cytogenetically normal AML patients younger than 60 years was a major advance in the last decade [3].During recent years, considerable progress has been made in understanding disease pathogenesis, and in development of diagnostic assays and novel therapies[4].

What is new in the 2016 Revision to the World Health Organization classification of $\mathrm{AML}$ and related Neoplasms

\section{AML with Balanced Genomic Rearrangements or Fusion Genes}

APL with cryptic PML-RARA fusion or complex cytogenetic rearrangements other than $t(15 ; 17)(q 24.1 ; q 21.2)$ is renamed APL with PML-RARA. AML with mutated NPM1 and AML with biallelic mutations of CEBPA have become full entities. New provisional entities AML with mutated RUNX1" (excluding cases with myelodysplasia-related changes) and AML with BCR-ABL1 are introduced. Minor updates in gene names (such as the change from MLL to KMT2A) are included as well as the recognition that the $\operatorname{inv}(3)(\mathrm{q} 21.3 \mathrm{q} 26.2)$ or $\mathrm{t}(3 ; 3)(\mathrm{q} 21.3 ; \mathrm{q} 26.2)$ does not represent a fusion gene[5], but repositioning of a GATA2 enhancer element leading to overexpression of the MECOM (EVI1) gene and to haploinsufficiency of GATA2[4].

Why RUNX1-Mutated Subtype was created in the New Classification?

The RUNX1-mutated subtype of AML was exclusive of recurrent genetic abnormalities recognized by the WHO and has adverse clinical outcomes[3].RUNX1 mutations are frequently observed together with FLT3-ITD, FLT3-TKD, and MLL-PTD as well as, with mutations in other AML driver genes (ASXL1, CEBPA, DNMT3A, NRAS, KIT, IDH1, IDH2, WT1). Interestingly, RUNX1 and NPM1 mutations seem to be mutually exclusive[6].

How to differentiate between AML with BCR-ABL1 and chronic Myeloid Leukemia Blast Phase?

Preliminary data suggest that deletion of antigen receptor genes (immunoglobulin heavy chain and T-cell receptor),IKZF1, and/or CDKN2A may support a diagnosis of AML rather than chronic myeloid leukemia blast phase[4].

\section{AML with Myelodysplasia-Related Changes}

The presence of $50 \%$ or more dysplastic cells in at least 2 cell lines is defined as multilineage dysplasia[5]. Presence of multilineage dysplasia with NPM1 mutation or biallelic mutation 


\section{Cancer Therapy \& Oncology International Journal}

of CEBPA does not classify them as AML with myelodysplasiarelated changes[5]. Deletion $9 q$ was removed from the list because of its association with $t(8 ; 21)$, and its frequent occurrencein AML with NPM1 and biallelic CEBPA mutations[4].

\section{Therapy-Related Myeloid Neoplasms(t-MNs)}

The t-MNs may be further subdivided as therapy-related MDS or AML. A number of t-MN cases have been shown to have germ line mutations in cancer susceptibility genes[5].The presence of mutations in a spliceosomal gene (SF3B1, SRSF2, U2AF1, or ZRSR2), ASXL1, EZH2, BCOR, or STAG2 was >95\% specific for AML with an antecedent hematologic malignancy in a previous study[3].Myeloid sarcoma is listed separately in the classification[5]

\section{Myeloid proliferations of Down Syndrome}

It includes TAM and myeloid leukemia associated with Down syndrome. Both are characterized by GATA1 mutations and mutations of the JAK-STAT pathway, with additional mutations identified in the myeloid leukemia cases. Both are usually megakaryoblastic proliferations. TAM occurs at birth or within days of birth and resolves in 1 to 2 months and myeloid leukemia occurs usually in the first 3 years of life with or without prior TAM and persists if not treated. The myeloid neoplasms of Down syndrome are not subclassified into MDS or AML. They have a similar behavior independent of blast cell count [5].

\section{Acute Leukemias of Ambiguous Lineage (MPAL)}

Diagnosis requires expression of antigens of $>1$ lineage i.e.Presence of distinct blast populations of different lineages, or 1 blast population with expression of antigens of different lineages on the same cells, or a combination[4]. No new entities were defined within this subgroup. The small list of specific lineage markers useful for defining MPAL is unchanged[5].

Myeloid neoplasms with germ line predisposition" (synonyms: familial myeloid neoplasms; familial myelodysplastic syndromes/acute leukemias): A new category [4] that includes patients with germ line mutations in CEBPA, DDX41, RUNX1, ANKRD26, ETV6, and GATA2 among a host of conditions marked by BM failure syndromes, telomere dysfunction, and germ line mutations activating RAS signaling[3].

\section{AML, not otherwise Specified}

In the new classification, myeloblasts are always counted as a percentage of total marrow cells.The previous subcategory of acute erythroid leukemia, erythroid/myeloid type (defined as a case with $\geq 50 \%$ BM erythroid precursors and $\geq 20 \%$ myeloblasts among non erythroid cells) has been removed [5]. Blastic plasmacytoid dendritic cell neoplasm (BPDCN)harbors a gene expression profile similar to AML and has mutations in genes commonly altered in myeloid neoplasms such as TET2, ASXL1, RNA-splicing factors, and TP53. The cell of origin is believed to be a plasmacytoid dendritic cell. BPDCN is distinct from AML and garners its own category in the newest WHO classification. The balanced translocation $\mathrm{t}(3 ; 5)(\mathrm{q} 21 ; \mathrm{q} 31)$, is specific to BPDCN. Additionally, expression of the transcription factor TCF4 was recently shown to be a faithful diagnostic biomarker of BPDCN[3].

\section{AML Risk Status by Cytogenetics and Molecular Abnormalities}

In addition to the categories defined by the WHO, 3 new AML subgroups emerged: 1) AML with mutations in chromatin and/ or spliceosome genes, 2) AML with mutations in TP53 and/or aneuploidy [3], the majority ( $>60 \%$ ) of cases in this subgroup have complex karyotypes [2] (defined as 3 or more chromosomal abnormalities in the absence of 1 of the WHO-designated recurring translocations or inversions)[4], and 3) AML with IDH2R172 mutation. Patients in the "chromatin-spliceosome" or "TP53-aneuploidy" groups (which account for nearly one-third of AML) had adverse outcomes. Analysis of at least the 13 genes contained within these 2 groups will be important to evaluate in future studies[3].Genome profiling analysis of the AML subtypes revealed that these broad patterns of genomic instability are accompanied by very specific and ordered patterns of comutations[2].

The 2017 ELN genetic risk stratification system uses a 3-group classification (favorable, intermediate, and adverse) rather than the previous 4-group system[4]. Biallelic CEBPA mutations that lack FLT3 or NPM1mutations have favorable prognostic significance[3].The presence of coexisting chromosomal abnormalities with NPM1 or biallelic CEBPA mutations does not modify their prognostic effect[4].Several concurrent additional genetic alterations impacting the outcome of established genetic predictors have been recognized recently in AML[3].Patients with NPM1mutation and a low FLT3-ITD allelic ratio (FLT3ITDlow) $(<0.5)$ and patients with a NPM1 mutation but no FLT3ITD have a favorable outcome[4]. AML with wild-type NPM1 and a high FLT3-ITD allelic ratio (FLT3-ITDhigh) $(\geq 0.5)$ as well as RUNX1, ASXL1, TP53 mutations and monosomal karyotype have been placed in the adverse-risk group[4].Monosomal karyotype is defined as the presence of 1 single monosomy (excluding loss of $\mathrm{X}$ or $\mathrm{Y}$ ) in association with at least 1 additional monosomy or structural chromosome abnormality (excluding core-binding factor AML)[4].KIT mutations have adverse prognostic impact among patients with $\mathrm{t}(8 ; 21)$ or inv(16)/t(16;16) AML[3]. DNMT3A, IDH1, IDH2, or genes in the chromatin/spliceosome group other than ASXL1 and RUNX1, are not assigned to an ELN prognostic group [4]

\section{Conclusion}

According to 2017 ELN guidelines, the recommended genetic analysis in newly diagnosed AML include:Cytogenetics, screening for gene mutations (NPM, FLT3, CEBPA, TP53, RUNX1, ASXL1) and screening for gene rearrangements (PML-RARA, 


\section{Cancer Therapy \& Oncology International Journal}

CBFB-MYH11, RUNX1-RUNX1T1, BCR-ABL1, other fusion genes if available)[4].

\section{References}

1. DiNardo CD, Cortes JE (2016) Mutations in AML: prognostic and therapeutic implications. Hematology Am Soc Hematol Educ Program 2016(1): 348-355.

2. Moarii M, Papaemmanuil E (2017) Classification and risk assessment in AML: integrating cytogenetics and molecular profiling. ASH Education Book 2017(1): 37-44.

3. Taylor J, Xiao W, Abdel-Wahab 0 (2017) Diagnosis and classification of hematologic malignancies on the basis of genetics. Blood 130(4): 410-423.

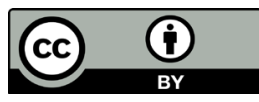

This work is licensed under Creative Commons Attribution 4.0 License

DOI: 10.19080/CTOIJ.2018.11.555809
4. Dohner H, Estey E, Grimwade D, Amadori S, Appelbaum FR, et al (2017) Lowenberg B, Bloomfield CD. Diagnosis and management of AML in adults: 2017 ELN recommendations from an international expert panel. Blood 129(4): 424-447.

5. Arber DA, Orazi A, Hasserjian R, Thiele J, Borowitz MJ, et al. (2016) The 2016 revision to the World Health Organization classification of myeloid neoplasms and acute leukemia. Blood 127(20): 2391-2405.

6. Sood R, Kamikubo Y, Liu P (2017) Role of RUNX1 in hematological malignancies. Blood 129(15): 2070-2082.

\section{Your next submission with Juniper Publishers will reach you the below assets}

- Quality Editorial service

- Swift Peer Review

- Reprints availability

- E-prints Service

- Manuscript Podcast for convenient understanding

- Global attainment for your research

- Manuscript accessibility in different formats

( Pdf, E-pub, Full Text, Audio)

- Unceasing customer service

Track the below URL for one-step submission https://juniperpublishers.com/online-submission.php 